\title{
Spiking neuromorphic chip learns entangled quantum states
}

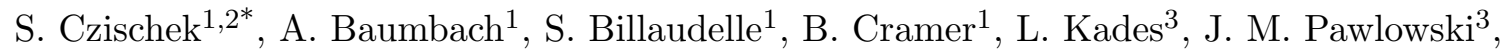 \\ M. K. Oberthaler ${ }^{1}$, J. Schemmel ${ }^{1}$, M. A. Petrovici ${ }^{4,1}$, T. Gasenzer ${ }^{1,3}$, M. Gärttner ${ }^{1,3,5}$ \\ 1 Kirchhoff-Institut für Physik, Ruprecht-Karls-Universität Heidelberg, Im Neuenheimer \\ Feld 227, 69120 Heidelberg, Germany \\ 2 Department of Physics and Astronomy, University of Waterloo, Ontario, N2L 3G1, Canada \\ 3 Institut für Theoretische Physik, Ruprecht-Karls-Universität Heidelberg, Philosophenweg \\ 16, 69120 Heidelberg, Germany \\ 4 Department of Physiology, University of Bern, 3012 Bern, Switzerland \\ 5 Physikalisches Institut, Universität Heidelberg, Im Neuenheimer Feld 226, 69120 \\ Heidelberg, Germany \\ * sczischek@uwaterloo.ca
}

October 27, 2021

\begin{abstract}
The approximation of quantum states with artificial neural networks has gained a lot of attention during the last years. Meanwhile, analog neuromorphic chips, inspired by structural and dynamical properties of the biological brain, show a high energy efficiency in running artificial neural-network architectures for the profit of generative applications. This encourages employing such hardware systems as platforms for simulations of quantum systems. Here we report on the realization of a prototype using the latest spike-based BrainScaleS hardware allowing us to represent few-qubit maximally entangled quantum states with high fidelities. Extracted Bell correlations for pure and mixed two-qubit states convey that non-classical features are captured by the analog hardware, demonstrating an important building block for simulating quantum systems with spiking neuromorphic chips.
\end{abstract}

\section{Contents}

1 Introduction $\quad 2$

2 Neuromorphic encoding of quantum states 2

3 Encoding an entangled Bell state 5

4 Learning performance 8

5 Deep and partially restricted networks 9

6 Conclusion 9 
A Implementation details of BrainScaleS-2 11

B Computation time benchmark for sampling from neural networks 14

C Representation of the Bell state

D Training algorithm

\begin{tabular}{ll} 
References & 19 \\
\hline
\end{tabular}

\section{Introduction}

As von-Neumann computers are rapidly approaching fundamental physical limitations of conventional semiconductor technology, a number of alternative computing architectures are currently being explored. Among them, neuromorphic devices [1,2], which take inspiration from the way the human brain works, hold promise of having a wide range of applications, in particular in machine learning and artificial intelligence [3 11]. Here we focus on using them as a sampling device to emulate measurement outcomes in quantum physics [12, which are inherently probabilistic in nature. The BrainScaleS neuromorphic system [11] is ideally suited for this task. The accelerated analog circuit dynamics and the inherently parallel nature of the neuromorphic substrate enable a rapid generation of samples which carries the potential of scaling benefits as compared to von-Neumann devices (App. A).

We use neuronal spikes (action potentials) to mark transitions between discrete states and thereby effectively carry out the sampling process. The all-or-nothing nature of spikes represents a blessing in disguise. On the one hand, it does have an apparent drawback by making the computation of gradients - and thus, training - more demanding than in classical deep neural networks [2]. On the other hand, it also allows us to use a spiking neuromorphic substrate in the first place, the speed-up of which we harness for efficient Hebbian learning [13.

Since any quantum state can be mapped to a probability distribution [14, 15], it can, in turn, be represented using networks of leaky integrate-and-fire (LIF) neurons [16 18. Here, we use the BrainScaleS-2 chip [11 as a physical substrate to emulate such networks. This mixed-signal neuromorphic platform is centered around an analog core: neuro-synaptic states are represented as voltages and currents in integrated electronic circuits and evolve in continuous time. Its configurable connectivity of neurons allows us to explore various different network topologies, including shallow, as well as deep and densely connected ones. With this substrate, we demonstrate an approximate representation of quantum states with spiking neural networks that is sufficiently precise for encoding genuine quantum correlations.

\section{Neuromorphic encoding of quantum states}

In classical machine learning, generative models based on artificial neural networks are used to encode and sample from probability distributions [13]. Similarly, spiking neural networks 

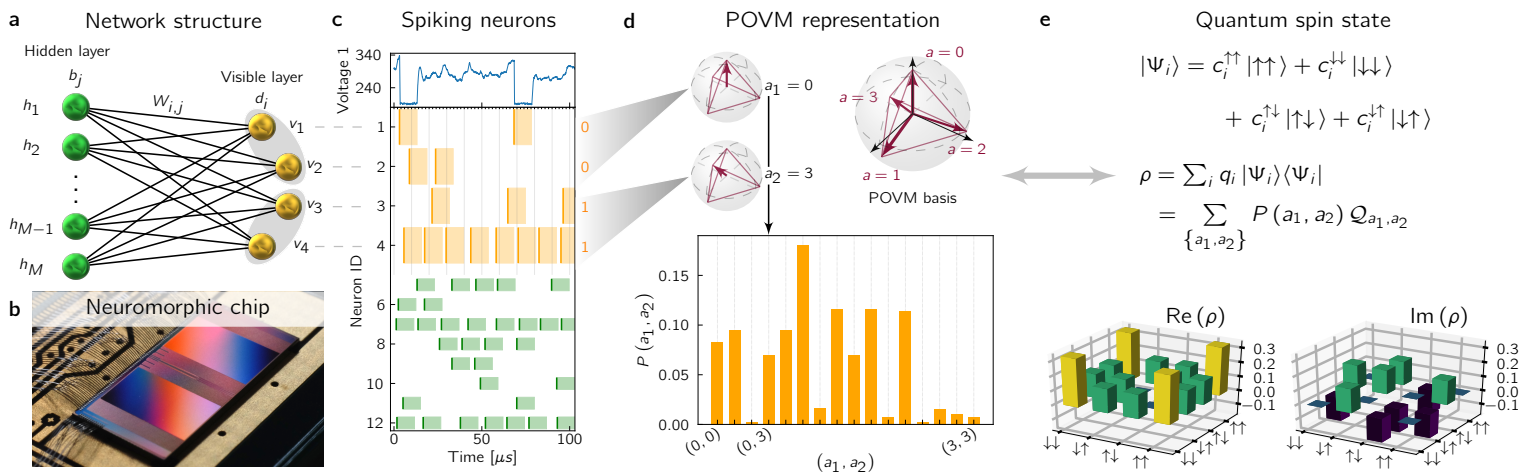

Figure 1: Neuromorphic representation of quantum states. a, Two-layer spiking network architecture with weight parameters $W_{i, j}$ between the visible (orange) and hidden (green) neurons and biases $d_{i}\left(b_{j}\right)$ for the binary visible (hidden) neurons. $\mathbf{b}$, Photograph of the BrainScaleS-2 chip used as a substrate for the experiments in this work. c, Dynamical evolution of the spiking network. Upper panel: membrane potential evolution of a single LIF neuron integrating synaptic input. Whenever the potential crosses a threshold a spike is generated and the potential is clamped to prevent immediate refiring (refractory period). Lower panels: Spikes (solid lines) for 4 visible (orange) and 8 hidden (green) neurons with associated $z=1$ time frames (shaded regions). The network state is observed periodically (gray lines showing only every fifth observation time for visibility reasons). Each observation results in a binary vector corresponding to a sample drawn from the underlying distribution. $\mathbf{d}$, The 4-state positive-operator-valued measure (POVM) representation of a qubit state can be encoded by a pair of visible neurons. A combination of $N$ such neuron pairs thus serves to represent an $N$-qubit system. The frequency of occurrence of neuron configurations drawn from a trained network encodes the POVM probability distribution of a quantum state (lower panel). e, Any quantum state can be represented as a density matrix $\rho$, which can be a statistical mixture of states $\left|\Psi_{i}\right\rangle$. For the example of two qubits shown here, the complex-valued entries of $\rho$ can be reconstructed linearly from the sampled probabilities $P\left(a_{1}, a_{2}\right)$. For the definition of the operators $\mathcal{Q}_{a_{1}, a_{2}}$, see App. C. 
can be viewed as approximating Markov-chain Monte-Carlo sampling, albeit with dynamics that differ fundamentally from standard statistical methods [19. Here, we encode quantum states using the hierarchical network architecture illustrated in Fig. 1 $1 \mathrm{a}$. The network consists of $N$ visible and $M$ hidden leaky integrate-and-fire (LIF) neurons arranged in a bipartite graph with a symmetric connectivity matrix. Such a network can be tuned to approximate the probability of the visible neurons to be in state $\boldsymbol{v}=\left(v_{1}, \ldots, v_{N}\right), v_{i} \in\{0,1\}$, as the marginal

$$
p(\boldsymbol{v} ; \mathcal{W})=\frac{1}{Z(\mathcal{W})} \sum_{\{\boldsymbol{h}\}} \exp [-E(\boldsymbol{v}, \boldsymbol{h} ; \mathcal{W})]
$$

over all hidden states $\boldsymbol{h}=\left(h_{1}, \ldots, h_{M}\right)$, where $h_{j} \in\{0,1\}$, of the joint Boltzmann distribution $p(\boldsymbol{v}, \boldsymbol{h} ; \mathcal{W})=\exp [-E(\boldsymbol{v}, \boldsymbol{h} ; \mathcal{W})][19]$. The network energy $E(\boldsymbol{v}, \boldsymbol{h} ; \mathcal{W})=-\sum_{i, j} v_{i} W_{i, j} h_{j}-$ $\sum_{i} v_{i} d_{i}-\sum_{j} h_{j} b_{j}$ depends on the set of network parameters $\mathcal{W}=(W, \boldsymbol{b}, \boldsymbol{d})$ including the weights $W_{i, j}$ and biases $b_{j}$ and $d_{i}$. The partition $\operatorname{sum} Z(\mathcal{W})=\sum_{\{\boldsymbol{v}, \boldsymbol{h}\}} p(\boldsymbol{v}, \boldsymbol{h} ; \mathcal{W})$ ensures normalization.

The BrainScaleS-2 system, depicted in Fig. 1 p, features 512 LIF neuron circuits interacting through a configurable weight matrix 11. Communication in a network of LIF neurons is realized via spikes. Whenever the membrane potential of a neuron, which is the result of a leaky integration of the synaptic input, exceeds a threshold, it sends a spike to the neurons connected to it (Fig. 1. , top panel). In the spike-based sampling framework, the refractory period $\tau_{\text {ref }}$ following a spike encodes the state $z=1$, while $z=0$ at all other times (Fig. 1 ;, lower panel). The stochasticity required for sampling is induced by adding a random component to the generation of spikes; for LIF networks, this can be ensured by sufficiently noisy membrane potentials 16, 18. To this end, we used on-chip sources to inject pseudo-Poisson spike trains into the network (see App. A).

As an experimental result, the BrainScaleS-2 chip returns a list of all spike times and associated neuron IDs. This information is sufficient to reconstruct the network state at any point in time. We estimated the distribution sampled by the network by observing its state at regular intervals, as visualized in Fig. 11k. To ensure an optimal estimate, the observation frequency needs to be at least $\left(\tau_{\text {ref }} / 2\right)^{-1}$ (see App. A). For our analysis, we used $\left(\tau_{\text {ref }} / 5\right)^{-1}$, thereby guaranteeing a large safety margin. The resulting binary configurations are collected in a histogram as shown in Fig. 11d.

A pure quantum state is described by a vector in Hilbert space and can be represented by a hermitian density matrix with complex entries. Density matrices can also encode mixed states and thus account for a possible coupling to an environment, which is relevant for a realistic description of experiments. Fig. 1e shows an example of a density matrix for a system of two spin-1/2 degrees of freedom (qubits) corresponding to a Hilbert-space dimension $d=4$. The corresponding probability distribution which we encode in our network is obtained from a so-called tomographically complete measurement 14 . Such a measurement has $d^{2}$ possible outcomes. Mathematically, these outcomes are represented by a set of operators $\left\{M_{\boldsymbol{a}}\right\}_{\boldsymbol{a}}$, forming a so-called positive-operator-valued measure (POVM). The density matrix can be reconstructed uniquely as $\rho=\sum_{\{\boldsymbol{a}\}} P(\boldsymbol{a}) \mathcal{Q}_{\boldsymbol{a}}$ from the probabilities $P(\boldsymbol{a})=\operatorname{Tr}\left[\rho M_{\boldsymbol{a}}\right]$ for obtaining outcome $\boldsymbol{a}$ according to Born's rule. The operators $\mathcal{Q}_{\boldsymbol{a}}$ are given by $\mathcal{Q}_{\boldsymbol{a}}=$ $\sum_{\left\{\boldsymbol{a}^{\prime}\right\}} T_{\boldsymbol{a}, \boldsymbol{a}^{\prime}}^{-1} M_{\boldsymbol{a}^{\prime}}$, with $T_{\boldsymbol{a}, \boldsymbol{a}^{\prime}}=\operatorname{Tr}\left[M_{\boldsymbol{a}} M_{\boldsymbol{a}^{\prime}}\right]$ [15]. In our two-qubit example (Fig. 11d) we chose $M_{\boldsymbol{a}}=M_{a_{1}} \otimes M_{a_{2}}$, where $M_{a_{i}}\left(a_{i}=0, \ldots, 3\right)$ are projection operators onto the single-qubit states represented as the four corners of a tetrahedron on the Bloch sphere. As each $a_{i}$ can take 
four different values, the encoding of the probabilities $P(\boldsymbol{a})$ by a spiking network is realized by representing each qubit by a pair of binary neurons in the visible layer (cf. gray shadings in Fig. 1 1 ). This results in the distribution $p^{*}(\boldsymbol{v})$ over the visible neurons (see App. C).

To approximate $p^{*}(\boldsymbol{v})$ through spike-based sampling, the parameters of the spiking network were adjusted in an iterative training procedure. We used the Kullback-Leibler divergence

$$
D_{\mathrm{KL}}\left(p^{*} \| p\right)=\sum_{\{\boldsymbol{v}\}} p^{*}(\boldsymbol{v}) \ln \left[\frac{p^{*}(\boldsymbol{v})}{p(\boldsymbol{v} ; \mathcal{W})}\right]
$$

to measure the quality of the sampled marginal $p(\boldsymbol{v} ; \mathcal{W})$. In each training epoch, the synaptic weights were updated along the gradient of the $D_{\mathrm{KL}}$ (see App. D):

$$
\Delta W_{i, j} \propto\left\langle v_{i} h_{j}\right\rangle_{\text {target }}-\left\langle v_{i} h_{j}\right\rangle_{\text {model }} .
$$

Pairwise correlations $\left\langle v_{i} h_{j}\right\rangle_{\text {model }}$ in the network were directly estimated from the sampled distribution $p(\boldsymbol{v}, \boldsymbol{h} ; \mathcal{W})$. Target correlations were also obtained from the sampled distribution by renormalization to the target marginal distribution:

$$
\left\langle v_{i} h_{j}\right\rangle_{\text {target }}=\left\langle\frac{p^{*}(\boldsymbol{v})}{p(\boldsymbol{v} ; \mathcal{W})} v_{i} h_{j}\right\rangle_{p(\boldsymbol{v}, \boldsymbol{h} ; \mathcal{W})}
$$

A similar scheme was used for the neuronal biases $b_{j}$ and $d_{i}$. This otherwise prohibitively compute-intensive method was made possible by the accelerated hardware dynamics and allows a much better approximation of the $D_{\mathrm{KL}}$ gradient than the more conventional contrastive divergence update scheme [13. Moreover, it does not rely on layer-wise conditional independence, allowing the exploration of network topologies other than bipartite graphs.

\section{Encoding an entangled Bell state}

To demonstrate that a spiking neural network can learn to represent entangled quantum states we focus on a maximally entangled two-qubit state, the Bell state $\left|\Psi^{+}\right\rangle=(|\uparrow \uparrow\rangle+|\downarrow \downarrow\rangle) / \sqrt{2}$. This state is a prototypical example exhibiting quantum mechanical correlations 20,21. We trained a network of four visible and 20 hidden neurons to encode the POVM probability distribution corresponding to $\rho_{\mathrm{B}}=\left|\Psi^{+}\right\rangle\left\langle\Psi^{+}\right|$. For calculating the weight updates in each epoch of the training procedure, as well as for evaluating expectation values, we drew 125000 samples of neuron states. This number is sufficient for the saturation of the $D_{\mathrm{KL}}$ as can be seen in Fig. 3b and was used for all experiments, if not specified otherwise.

To characterize the learned quantum state, we used the observable $\mathcal{B}(\Theta)$, which can signal genuine quantum correlations and is experimentally accessible via measurements as illustrated and defined in Fig. 2a: The two qubits are distributed to two parties who independently perform one of two possible measurements on their respective qubit. We choose the standard parametrization of the different measurements by a single angle $\Theta$. For a Bell state this procedure yields correlations violating the inequality $|\mathcal{B}(\Theta)| \leq 2$, which is obeyed by classical systems 21]. At $\Theta=\pi / 4$ this inequality is maximally violated for the Bell state $\rho_{\mathrm{B}}$ and thus yields an experimentally accessible witness for Bell correlations 20,22 .

The correlations encoded by the trained spiking network clearly exceed the classicality bound $|\mathcal{B}(\Theta)|=2$ (red points in Fig. 2 $\mathrm{b}$ ) and are in agreement with their exact $\Theta$-dependence 

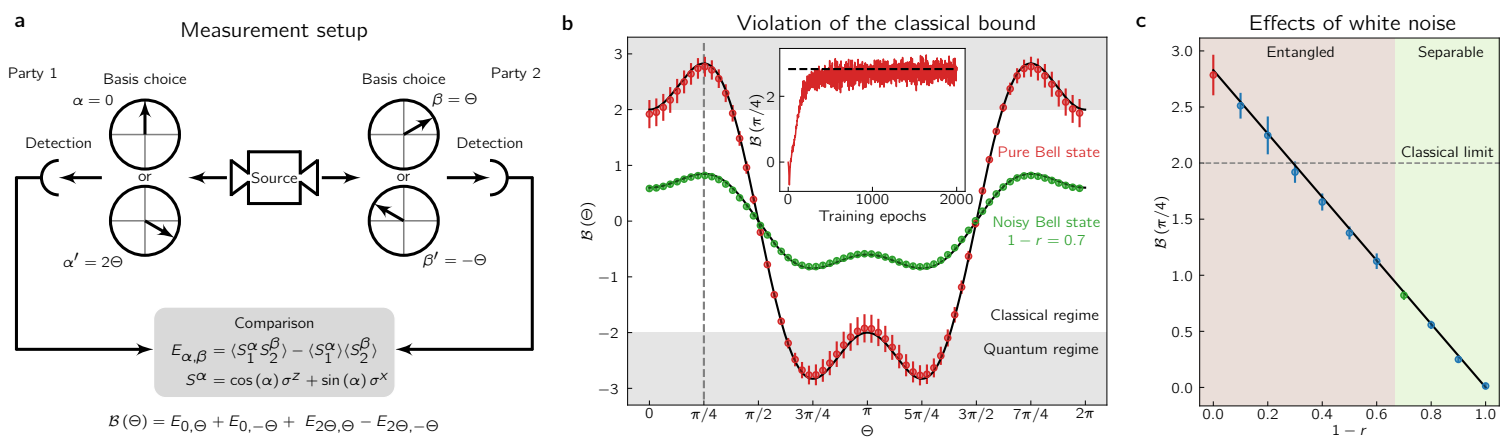

Figure 2: Encoding Bell states and Werner states. a, Illustration of a typical Bell-test scenario. Two correlated qubits emerging from a source are distributed between two parties. Each of the parties is allowed to choose between two different measurements each characterized by a single common angle $\Theta$. The measurement outcomes indicate genuine quantum correlations if the combination $\mathcal{B}(\Theta)$ of the correlations violates the inequality $|\mathcal{B}(\Theta)| \leq 2$ obeyed by classical states. $\mathbf{b}$, Observable $\mathcal{B}(\Theta)$ evaluated on the learned encoding of the Bell state $\rho_{\mathrm{B}}=\left|\Psi^{+}\right\rangle\left\langle\Psi^{+}\right|$on the neuromorphic hardware, with $M=20$ hidden neurons. Red symbols depict the observable for different angles $\Theta$, averaged over the last 200 training epochs, where errorbars here and in the following denote the standard deviation. Note that these data points have been obtained from the same trained network and the same set of neuron states sampled from it by evaluating the observable for different angles $\Theta$ on this sample set. Werner states $\rho_{\mathrm{W}}=r \rho_{\mathrm{B}}+(1-r) \mathbb{1} / 4$ are obtained by adding white noise to the pure Bell state. Green points correspond to $r=0.3$. In both cases, the data capture the exact values (black lines) well, including the violation of the classical bound in the pure case $r=1$. The inset shows the evolution of the Bell-correlation witness $\mathcal{B}(\Theta=\pi / 4$ ) during training (red line) and the convergence towards the expected value (black dashed line). c, Bell-correlation witness $\mathcal{B}(\Theta=\pi / 4)$ for a Werner state as a function of the noise strength $1-r$. The exact solution (black line) is captured well for both entangled and separable states. 


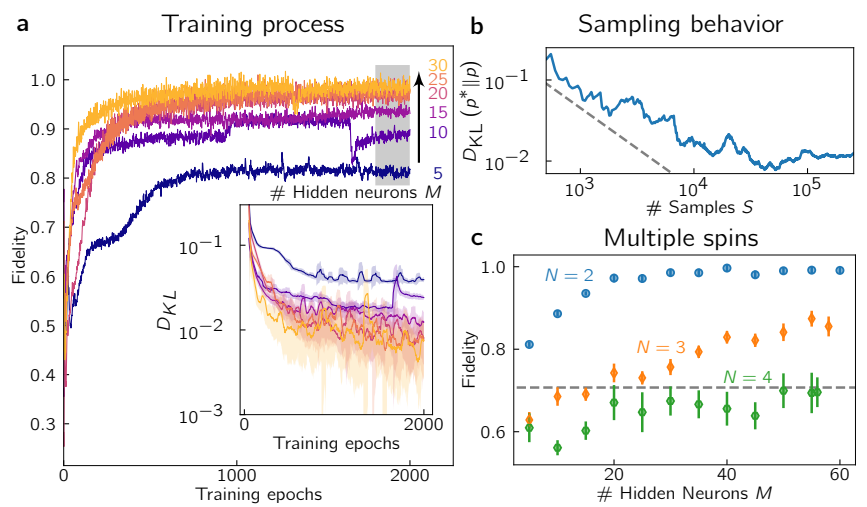

Figure 3: Training performance. a, Dynamics of the learning procedure for the pure Bell state $\rho_{\mathrm{B}}$. The quality of the network-encoded state is measured by the quantum fidelity, Eq. (5) (main frame), and by the Kullback-Leibler divergence, Eq. (2) (inset), for different numbers of hidden neurons. For better visibility, the running average over 50 epochs is shown in the inset as solid lines, with the shaded areas indicating the corresponding standard deviation. b, Kullback-Leibler divergence in a fixed trained network with $M=20$ hidden neurons as a function of the number of samples drawn. The dashed line shows the expected trend for exact sampling from the target distribution. c, Quantum fidelity as a function of the number of hidden neurons for GHZ states $|\Psi\rangle=\left(|\uparrow\rangle^{\otimes N}+|\downarrow\rangle^{\otimes N}\right) / \sqrt{2}$ with $N=2$ (Bell state), 3, and 4 qubits. We show the averages over 200 training epochs after convergence (gray shaded area in a). The dashed line shows the bound for genuine $N$-partite entanglement.

(black line). The inset shows how the Bell correlation witness $\mathcal{B}(\Theta=\pi / 4)$ develops during the training, converging after less than 1000 iterations.

To illustrate the generality of our neuromorphic encoding scheme we consider mixed quantum states by adding white noise to the pure Bell state resulting in the Werner state $\rho_{\mathrm{W}}=r \rho_{\mathrm{B}}+(1-r) \mathbb{1} / 4$ with noise strength $0 \leq 1-r \leq 1$ [23]. Increasing the noise reduces $|\mathcal{B}(\Theta)|$ and eventually confines it within the classical regime (cf. green data in Fig. 2p). For $1-r>1 / \sqrt{2}$ the Bell correlation witness fails to detect entanglement, and for $1-r>2 / 3$ the state becomes separable (unentangled). The resulting mixed states are faithfully represented by our system for any value of $r$ as shown in Fig. 2r. The fluctuations in the experimental data decrease with increasing noise contribution, allowing a more accurate learning of mixed states. This counterintuitive effect is due to additional noise leading to an increase in entropy, which is synonymous with sampling from more uniform distributions. These, in turn, are realized by weaker weights, thus decreasing the influence of imperfect synaptic interactions in the neuromorphic substrate. 


\section{Learning performance}

We analyzed in detail the convergence of the learning algorithm using the classical KullbackLeibler divergence $D_{\mathrm{KL}}$ as defined in Eq. (2). In addition, we use the quantum fidelity

$$
\mathcal{F}\left(\rho_{\mathrm{B}}, \rho_{\mathrm{N}}\right)=\operatorname{Tr}\left[\sqrt{\sqrt{\rho_{\mathrm{B}}} \rho_{\mathrm{N}} \sqrt{\rho_{\mathrm{B}}}}\right]
$$

to quantify the distance between the target state $\rho_{\mathrm{B}}$ and the network-encoded state $\rho_{\mathrm{N}}$, which, for pure states, reduces to the state overlap. As shown in Fig. 3 a, the learning converges after 1000 training epochs. Increasing the number of hidden neurons we find that the fidelity reaches $\approx 98 \%$ (correspondingly $D_{\mathrm{KL}} \lesssim 10^{-2}$ ) for $M \gtrsim 20$ hidden neurons. The limited reachable fidelity is a result of many different factors of the physical implementation of the spiking neural network on the BrainScaleS-2 platform. The synaptic connections are implemented with 6bit resolution, limiting the achievable precision of approximating the probability distribution. Also, uncontrolled environmental changes such as temperature variations or host-to-system effects influence the performance of the hardware. This manifests in the jumps of fidelity occurring during learning, as well as in strong noise in the fidelity after the learning process has saturated, as can be seen in Fig. 33. These instabilities exceed the anticipated noise level due to finite sample statistics used for evaluating observables and calculating gradients in each epoch. These factors degrade the correspondence between the model assumption underlying the employed learning rule and the actual dynamics of the hardware. Many of the issues mentioned above can be resolved in future hardware generations.

To ensure that the learning performance is not limited by finite sample statistics, we evaluated the Kullback-Leibler divergence as a function of the number of samples in a trained network with fixed network parameters. Figure 3 b shows the expected convergence towards a minimum value determined by the quality with which the spiking network approximates the POVM distribution. Typically, for $>10^{5}$ samples the statistical error is negligible compared to the errors due to hardware noise and limited representational power of the network, causing the saturation of the DKL observed in Fig. 3b. This justifies our choice of training with 125000 samples per epoch.

Having demonstrated high-fidelity emulation of two-qubit entangled states, we investigated whether states of multiple qubits can also be encoded by our spiking sampling network. Figure 3 c shows the fidelity achieved in learning Greenberger-Horne-Zeilinger (GHZ) states [24], i. e. $N$-qubit generalizations of a Bell state, as a function of the number of hidden neurons $\bar{M}$. The underlying probability distribution covers a larger state space of the visible neurons, requiring us to increase the number of samples to 225000 to reach convergence in the $D_{\mathrm{KL}}$. In all cases the fidelity of the learned state to the perfect GHZ state increases with $M$, reaching values of close to $90 \%$ and about $70 \%$ for three and four qubits, respectively. As layered network architectures are known to require a large number of neurons for representing GHZ states [15], we assume that larger chip sizes will allow to increase these values further. Note that a GHZ-state fidelity above $\mathcal{F}=1 / \sqrt{2} \approx 70 \%$ means that the state exhibits genuine $N$-partite entanglement (cf. dashed line in Fig. 3r) 25]. 

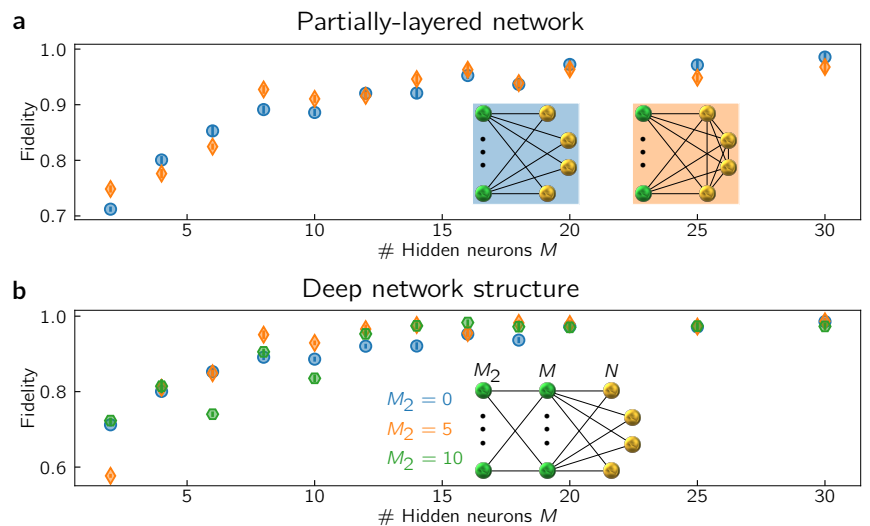

Figure 4: Extending the network architecture. a, Fidelity between network-encoded and perfect Bell state, Eq. (5), as a function of the number of hidden neurons for strictly layered network (blue) and an architecture with additional connections between the visible neurons (orange). b, Quantum fidelity for states encoded in a deep network architecture with a second hidden layer containing 5 (orange) and 10 (green) neurons compared to the restricted two-layer network (blue).

\section{$5 \quad$ Deep and partially restricted networks}

Our flexible learning scheme allows the training of network architectures beyond simple bipartite graphs. To explore network architectures with potentially larger representational power we added connections between the visible neurons, resulting in a more densely connected network. Figure 4 a shows that a Bell state can be encoded successfully with this architecture, reaching similar fidelities as the two-layer fully restricted spiking network. We also explored deeper network architectures by adding an additional hidden layer, see Fig. 4b. Again, the Bell state was learned successfully reaching similar fidelities as in the bipartite case. We note that the learning performance is not monotonic at small $M$ for $M_{2}=10$ neurons in the second hidden layer. This is expected, since the intermediate layer constitutes an information bottleneck towards the visible layer, which makes learning more difficult. Therefore, the greater representational power offered by additional depth 26 does not necessarily translate into a higher fidelity for $M<M_{2}$. The overall non-monotonic dependence of the fidelity on the number of hidden neurons is caused by hardware noise leading to fluctuating training performance.

The fact that the learning performance does not improve when using different architectures indicates that the reachable fidelity is currently limited by technical imperfections rather than the representational power of the ansatz. Larger-scale systems may be able to exploit the greater representational power of these deeper and more complex architectures.

\section{Conclusion}

We have shown that a spiking neural network implemented on a neuromorphic chip can approximate entangled quantum states of few particles with high quantum fidelity. In particular 
non-classical Bell correlations can be encoded faithfully, demonstrating that intrinsic quantum features can be captured by a classical spiking network.

The fidelities and system sizes achieved in this first study on neuromorphic quantum state encoding should be regarded as a proof of principle. The experienced restrictions are mainly technical in nature and can be improved in future generations of spiking neuromorphic devices. Specifically for the BrainScaleS-2 system, both the hardware and its surrounding software framework are in an ongoing maturation process. The size and fidelity of the approximated quantum states can be significantly improved upon by optimizing the usage of hardware realestate, the signal-to-noise ratio of the analog circuitry and the calibration of the chip. Judging from the current pace of progress in neuromorphic engineering, significantly larger systems, both digital and analog, can be expected to become available in the near future [1].

Furthermore, runtime improvements are anticipated, as the current bottleneck is the calculation of the weight updates of the network parameters, which is done "offline" on a conventional computer and only the sampling itself is performed on the chip (see App. A). Using the on-chip plasticity processor to update synaptic weights has the potential of drastically reducing the training time by removing the cumbersome chip-host loop [27.

One key advantage of this neuromorphic system as compared with simulated generative models is that scaling to larger network sizes does not increase the time needed to collect a desired number of samples. We illustrate this property by comparing the sampling time on the neuromorphic chip with a CPU implementation in App. B showing a gain through neuromorphic sampling already at moderate system sizes. Given the efficient learnability [28] and representability of important classes of quantum states 29 31, and the availability of sampling schemes for neuromorphic devices [32,33], we thus expect favorable scaling properties for our approach. Thus our work opens up a path towards applications of neuromorphic hardware in quantum many-body physics.

\section{Acknowledgments}

We are indebted to the late K. Meier who envisioned and championed the BrainScaleS system and made seminal contributions to this endeavor. We thank A. Kungl for discussions and the Electronic Vision(s) group, in particular E. C. Müller, C. Mauch, Y. Stradmann, P. Spilger, J. Weis, and A. Emmel, for maintaining and providing access to the BrainScaleS-2 system and for technical support.

Author contributions S.C. and A.B. carried out the experiment, analysed the data and wrote the paper. S.B., B.C., and A.B. configured the neuromorphic hardware and provided the software interface to access it. S.C., M.G., and T.G. developed the theory and designed the neuromorphic encoding scheme. All authors contributed to interpreting the data and writing the manuscript.

Funding Information This work is supported by the Deutsche Forschungsgemeinschaft (DFG, German Research Foundation) under Germany's Excellence Strategy EXC 2181/1 390900948 (the Heidelberg STRUCTURES Excellence Cluster), by the DFG - project-ID 273811115 - SFB 1225 (ISOQUANT), by the European Union 7th and Horizon-2020 Framework Programmes, through the ERC Advanced Grant EntangleGen (Project-ID 694561) and 
under grant agreements 604102, 720270, 785907, 945539 (HBP), and by the Manfred Stärk Foundation.

\section{A Implementation details of BrainScaleS-2}

The BrainScaleS-2 system is a mixed-signal neuromorphic platform. Its analog core is composed of neuron and synapse circuits with inherent time constants of the order of microseconds. An application-specific integrated circuit (ASIC) for the BrainScaleS-2 system features 512 neuron circuits, which emulate the adaptive exponential integrate-and-fire model. These individual compartments can be wired to resemble more complex structured neurons. An on-chip analog parameter memory as well as integrated static random-access memory (SRAM) cells allow us to individually configure and optimize the dynamics of each circuit. Each neuron integrates input from 256 dedicated synapses, which carry a 6-bit weight and can be either excitatory or inhibitory.

The analog core is accompanied by supporting logic, including circuitry for communication and configuration. Further functionality is provided by high-bandwidth spike sources, which can emit either regular or Poissonian spike trains of configurable frequency. A routing module allows mixing these spikes with external stimuli and recurrent events. It allows, in combination with in-synapse event filtering, the implementation of arbitrary network topologies.

Custom embedded processors allow the modification of the entire configuration space during the runtime of an experiment. Tightly coupled to the synaptic arrays, they allow the efficient and flexible implementation of learning rules based on observables such as neuronal potentials, firing rates, and synaptic correlations.

A network of leaky integrate-and-fire (LIF) neurons can implement a sampling spiking network (SSN) if the neurons are under stochastic noise influence, their membrane time constant is sufficiently small and the synaptic and refractory time constants roughly match [18]. A system-specific calibration is required to configure the analog core of BrainScaleS-2, shown in Fig. 5 a according to these requirements. For ease of implementation we use a simple routing scheme in which the on-chip network looks like 128 unique sources which can be arbitrarily connected. This allows the association of each of the 128 synapse drivers with one spike source while using the double line to implement signed synapses (cf. Fig. $5 \mathrm{~b}$ ).

The stochastic input spikes are generated via two of the eight on-chip linear shift registers (LSFRs). We assign the spike source IDs 0-63 to the network neurons and split the spike trains from the LSFRs among the IDs 64-127. For networks smaller than 64 neurons, the upper part of (0-63) remains unused. Again simplifying the implementation we use the first half of the noise IDs (64-95) as excitatory and the second half (96-127) as inhibitory sources (cf. Fig. 5c lower part). This scheme allows in principle all-to-all connectivity within the network. Choosing to use a layered network structure results in a block structure of the upper part of the synapse array (cf. Fig. 5. 5 ).

Each sampling neuron is connected to 5 randomly chosen excitatory and 5 randomly chosen inhibitory noise sources. This introduces correlations between neurons even without synaptic connections, but in general does not hinder training [16,34. Synaptic connections on BrainScaleS-2 are 6-bit-valued circuits. The dynamical impact of a single network spike (used to mediate the stochastic response of the receiving sampling unit) onto another neuron is given by its own strength relative to the total strength of the input provided by the background 

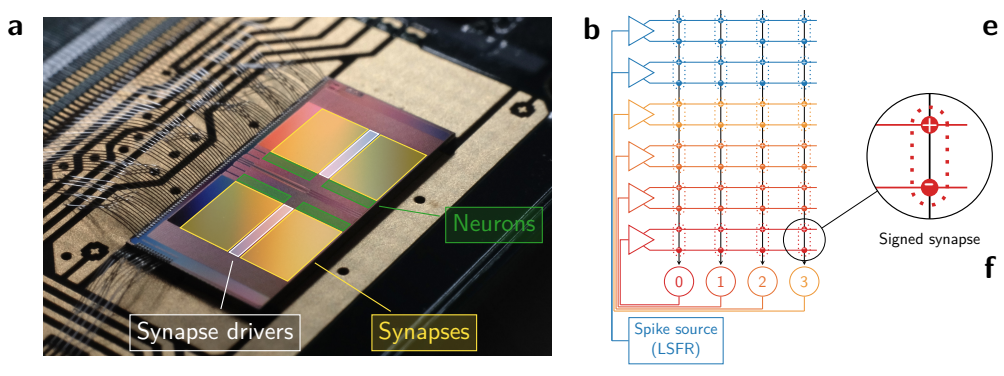

C

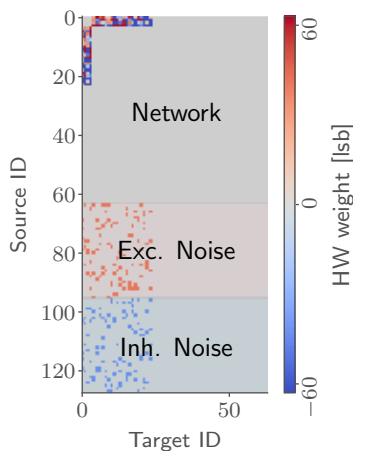

d

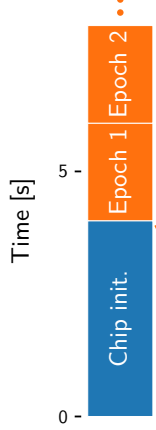

$2-$

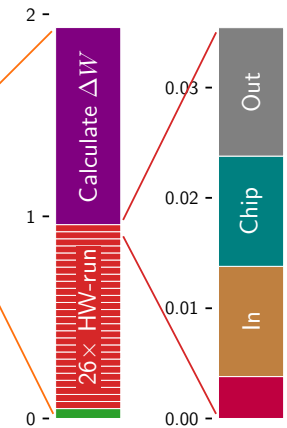

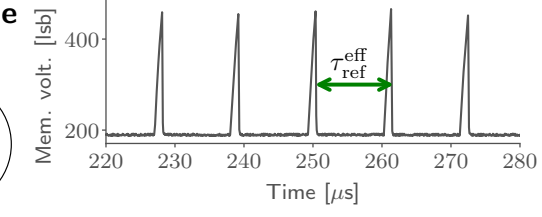

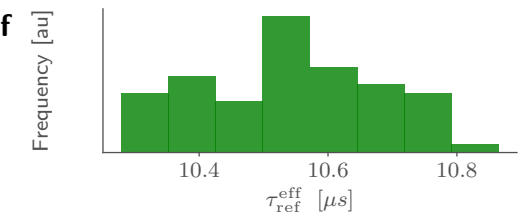

g

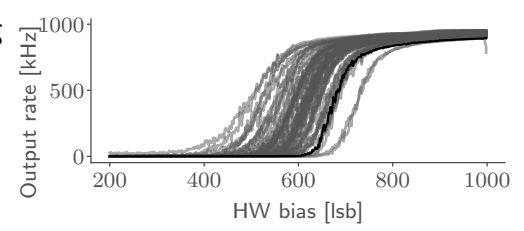

h

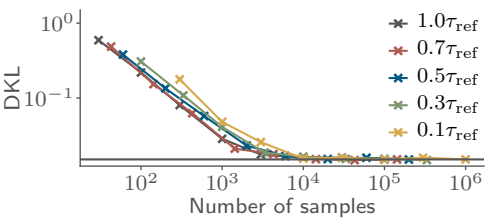

Figure 5: Details of the BrainScaleS-2 neuromorphic chip. a, Photograph of the BrainScaleS-2 chip with circuits of $4 \times 128$ AdEx-LIF neurons (green), $2 \times 2 \times 128$ synapse drivers (white) and 4 synapse arrays with $256 \times 128$ synapses (yellow). b, Routing schematic used to implement the sampling spiking network. Each synapse driver projects to two synapse rows in order to allow signed synapses. c, Utilized logical connectivity matrix projecting onto the 64 neurons used. Network (neuron-to-neuron) connections are truncated at index 24 (4 visible and 20 hidden) and intra-layer connections are not used. Each neuron receives noise input from 5 excitatory (64-95) and 5 inhibitory (96-127) sources, generated by one on-chip LSFR each. Each connection selects the appropriate synapse row depending on its sign (cf. b) d, Time usage across a training experiment. The initial configuration (blue) of the chip is comparable to a single epoch (orange). Each epoch consists of a parameter update (green), 26 sampling runs (red) and the update calculation (purple). Each hardware run consist of the construction of the playback program (ruby), the initial buffering on the FPGA (brown), the actual chip runtime (turquoise) and the readout to the host (grey). e, Membrane trace of an exemplary neuron at the high-bias end. $\tau_{\text {ref }}^{\text {eff }}$ is the inter spike interval. f, Histogram of measured $\tau_{\text {ref }}^{\text {eff }}$. Variations are due to the analog nature of the system. g, Activation functions as a function of the leak potential under noise input of the 64 neurons used. $\tau_{\text {ref }}^{\text {eff }}$ is estimated by the output frequency at the high-bias end. $\mathbf{h}$, Sampling performance as a number of samples, rather than execution time for different sampling time deltas $d t$. More than two samples per refractory time $\tau_{\text {ref }}^{\text {eff }} \approx 10 \mu$ s increase the Kullback-Leibler divergence as the samples are not independent. 
sources. The latter defines the transfer function and thereby the excitability of the neurons (cf. Fig. 5g). Choosing the noise parameters (weight and number of sources) is done such as to attain the competing goals of allowing the network neurons to drive each other significantly while allowing for small weight changes within the 6-bit resolution limit. The particular choice is, in general, problem dependent.

Having chosen the noise parameters, the sampling interface of BrainScaleS-2 becomes a black box that requires a weight (6-bit) matrix and a (10-bit) bias vector and returns a set of spike trains. Neurons are assigned a state of $z=1$ at time $t$ if they emitted a

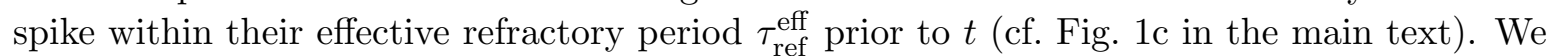
determine $\tau_{\text {ref }}^{\text {eff }}$ by setting the leak potential of the neurons to its maximum value and measuring the resulting inter-spike intervals (cf. Fig. 5e). The effective refractory time consists of the clamped part which is digitally driven and therefore does not vary between different neurons and the drift part back to the spiking threshold in the end. Due to the circuit variability (e.g. different membrane time constants) of the analog circuits we see some modest variation in $\tau_{\text {ref }}^{\text {eff }}$ (cf. Fig. 5 f). Using the measured $\tau_{\text {ref }}^{\text {eff }}$ we assign a state every $2 \mu$ s and use the set of these states for the evaluation and the update calculation.

Figure Fig. 5h demonstrates the correctness of an approximated distribution for a simulated sampling spiking network (using [35]) as a function of the number of samples for different state assign times $d t$ (cf. Fig. 1 in the main text). For more than two samples per refractory period $\tau_{\text {ref }}$ the number of samples required to achieve a given performance level increases due to the correlated states as expected from the Nyquist-Shannon theorem. Both the noise parameters and the sample frequency were chosen such that they enable sufficiently accurate sampling, but without performing an exhaustive optimization.

As discussed above, a chip-specific calibration is required but can be reused for each training. For each experiment the chip needs to be initialized (blue period in Fig. 5d) once. This ensures that the correct calibration is loaded and the routing is configured correctly before the training iterations (orange period in Fig. 5d) can start. After the initialization only the synapse array (weights) and the leak potentials of the neurons (biases) are reconfigured once per epoch (green period in Fig. 5d). Each training epoch consists of 26 sampling runs (red section in Fig. 5d) and a single calculation of the parameter update (purple in Fig. 5d). In each hardware run we build a program for the FPGA to execute (dark red in Fig. $5 \mathrm{~d}$ ), transfer it to the FPGA with some initial buffering (yellow in Fig. $5 \mathrm{~d}$ ) in order to compensate for network latencies, perform the actual execution on chip (light blue in Fig. 5d) and transfer the spikes back to the host computer (grey in Fig. 5d).

In total, an epoch takes about $1.9 \mathrm{~s}$ of which roughly half is spent in the sampling and the other half is used to calculate the parameter updates. While some time was spent to improve performance, both parts can still be optimized. For example the gradient calculation is implemented in Python and most of the sampling time is spent buffering and reading back the results. The actual hardware runtime is only $30 \%$ of the time marked as $H W$-run in Fig. 5d. Using a more complex routing setup an increase to at least 256-spike sources is possible and since BrainScaleS-2 is a physical system the runtime of the hardware part is not affected by the network size. 


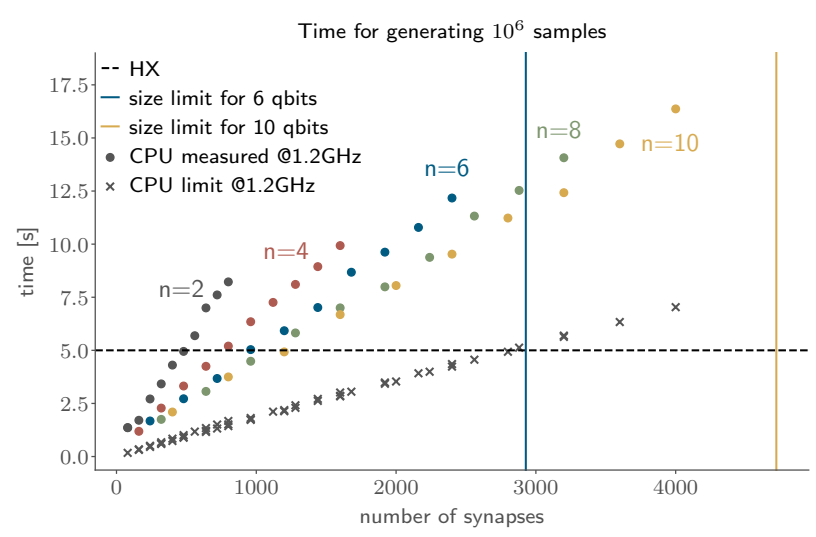

Figure 6: Measured (dots) and estimated (crosses) sampling times for the generation of a million samples, for different quantum system size $(N=2,4,6,8,10$ spins, colours $)$ and hidden layer sizes $(M=20,40,60,80,100,120,140,160,180,200)$ on a Intel Xeon E5-2630v4 compared to the constant runtime of the BrainScaleS-2 system (horizontal line). The software time estimation assumes one FLOP per clock cycle and one FLOP required per synaptic interaction, bias and state assignment (see text). The number of neurons on BrainScaleS-2 is limited to $(2 N)+M<256$ which limits the implementable system size (vertical lines, for 6 spins and 244 hidden neurons and 10 spins and 236 hidden neurons).

\section{B Computation time benchmark for sampling from neural networks}

In this section, we provide a speed comparison between the BrainScaleS-2 neuromorphic chip and a $\mathrm{C}++$-implemented software solution to the sampling from binary Boltzmann machines. The software implements standard Gibbs sampling, i.e. it sequentially calculates the "membrane potential" $u_{i}=b_{i}+\sum_{i} W_{k i} z_{i}$ for each neuron and assigns a new state $z_{i}=1$ with probability $\sigma\left(u_{i}\right)=\frac{1}{1+\exp -u_{i}}$ and $z_{i}=0$ otherwise. This implementation, while fairly optimized in single-thread performance, does not take into account the potential parallelism of a layered structure. Since the simulator is optimized for large-scale systems it drops all terms with $W_{k i}=0$, at the price of an additional indirection. The sum now runs over a list of indices which is harder to optimize than a simple sequential iteration. We executed this on the bwForCluster NEMO cluster [36] which uses Intel Xeon E5-2630v4 (Broadwell) CPUs.

Generating a new state requires the update of all neurons, and each update of a single neuron requires the calculation of $u_{i}$ plus a comparison with a random number for the probabilistic update. For the architecture used in the main manuscript, i.e. layered networks with $2 N$ visible and $M$ hidden neurons and assuming a perfect implementation without additional cost for memory accesses, generating a new update takes $2(2 N) M$ evaluations and additions of the term $W_{k i} z_{i}$, besides $(2 N)+M$ additions of $b_{i}$, and $(2 N)+M$ comparisons to a random number. Assuming further that each of these steps takes one clock cycle, we can estimate the expected time required.

In order to reduce the impact of the initialization of the software sampler (loading of the network configuration and initialization) we measure the time to generate $10^{6}$ samples. We note that the number of operations per update is dominated by the number of connections 
(synapses) 2(2N)M. As such, the time required scales linearly in the number of hidden units only for a fixed number of visible units, which is given by the size of the physical system (cf. Fig. 6).

On the other hand, the BrainScaleS-2 implementation, due to its inherently parallel architecture, requires a sample generation time that is independent of the size of the sampled network. With $\tau_{\text {ref }} / 2=5 \mu$ s per sample (cf. Fig. $5 \mathrm{~h}$ ), this leads to a constant time of $5 \mathrm{~s}$. This constant scaling is only true if the network fits onto the system (up to 256 sampling neurons). Since the number of visible neurons is given by the size of the physical system that is represented ( $N$ spins), larger physical systems give a greater speedup. Already for the case of 8 spins (16 visible units and 180 hidden units) the fixed runtime of the BrainScaleS-2 system is exceeded by our estimation from the idealized software estimate (cf. Fig. 6). Larger system sizes will skew this comparison further to favor of BrainScaleS-2, which can even implement more densely connected network topologies without incurring a performance penalty. We also note that the BrainScaleS-2 chip requires less than $500 \mathrm{~mW}$ [37, 38], while the Intel Xeon E5-2630v4 has a thermal design power (TDP) of $85 \mathrm{~W}$ for 10 cores. As such BrainScaleS-2 is using comparable energy even for the smallest systems we implemented in the prototype system used in the main manuscript. While the system size at which the BrainScaleS-2 chip outperforms CPU implementations may shift to larger values when comparing to the fastest currently available CPUs, the fundamental difference in scaling behavior, i.e. constant v.s. linear, persists.

\section{Representation of the Bell state}

The Bell state, $\left|\Psi^{+}\right\rangle=1 / \sqrt{2}(|\uparrow \uparrow\rangle+|\downarrow \downarrow\rangle)$, is described by the density matrix

$$
\rho_{\mathrm{B}}=\frac{1}{2}\left[\begin{array}{llll}
1 & 0 & 0 & 1 \\
0 & 0 & 0 & 0 \\
0 & 0 & 0 & 0 \\
1 & 0 & 0 & 1
\end{array}\right]
$$

in the standard basis. To encode this state in a spiking neural network, we map it to a POVM probability distribution.

While several choices of POVM representations are possible, we here focus on the tetrahedral representation, where each measurement projects a single qubit onto one corner of a tetrahedron in the Bloch sphere [15]. The POVM elements $M_{a_{i}}$ for each qubit $i$ can hence be expressed in the form $M_{a_{i}}=\left(\mathbb{1}+\boldsymbol{s}_{a_{i}} \boldsymbol{\sigma}\right) / 4$, with Pauli operators $\boldsymbol{\sigma}=\left(\sigma^{x}, \sigma^{y}, \sigma^{z}\right)$ and $s_{a_{i}=0}=$ $(0,0,1), s_{a_{i}=1}=1 / 3(2 \sqrt{2}, 0,-1), s_{a_{i}=2}=1 / 3(-\sqrt{2}, \sqrt{6},-1), s_{a_{i}=3}=1 / 3(-\sqrt{2},-\sqrt{6},-1)$. The POVM elements thus take the form

$$
\begin{aligned}
& M_{a_{i}=0}=\frac{1}{2}\left[\begin{array}{ll}
1 & 0 \\
0 & 0
\end{array}\right], \quad M_{a_{i}=1}=\frac{1}{6}\left[\begin{array}{cc}
1 & \sqrt{2} \\
\sqrt{2} & 2
\end{array}\right], \\
& M_{a_{i}=2}=\frac{1}{12}\left[\begin{array}{cc}
2 & -\sqrt{2}-\mathrm{i} \sqrt{6} \\
-\sqrt{2}+\mathrm{i} \sqrt{6} & 4
\end{array}\right], \\
& M_{a_{i}=3}=\frac{1}{12}\left[\begin{array}{cc}
2 & -\sqrt{2}+\mathrm{i} \sqrt{6} \\
-\sqrt{2}-\mathrm{i} \sqrt{6} & 4
\end{array}\right] .
\end{aligned}
$$


With this, the POVM probability distribution of the Bell state, $P_{\mathrm{B}}\left(a_{1}, a_{2}\right)=\operatorname{Tr}\left[\rho_{\mathrm{B}} M_{a_{1}} \otimes M_{a_{2}}\right]$, evaluates to

$$
P_{\mathrm{B}}=\frac{1}{8}\left[\begin{array}{cccc}
1 & 1 / 3 & 1 / 3 & 1 / 3 \\
1 / 3 & 1 & 1 / 3 & 1 / 3 \\
1 / 3 & 1 / 3 & 1 / 3 & 1 \\
1 / 3 & 1 / 3 & 1 & 1 / 3
\end{array}\right]
$$

where columns correspond to the index $a_{1}$ and rows to the index $a_{2}$.

To reconstruct the density matrix from this probability distribution, the inverse of the full-system overlap matrix $T$ is needed, which can be constructed as the product of the singlequbit overlap matrices, $T=T_{1} \otimes T_{2}$. Each single-qubit overlap matrix consists of the elements $T_{a_{i}, a_{i}^{\prime}}=\operatorname{Tr}\left[M_{a_{i}} M_{a_{i}^{\prime}}\right]$. For the tetrahedral POVM the inverse $T_{i}^{-1}$ of the single-qubit overlap matrix takes the form

$$
T_{i}^{-1}=\left[\begin{array}{cccc}
5 & -1 & -1 & -1 \\
-1 & 5 & -1 & -1 \\
-1 & -1 & 5 & -1 \\
-1 & -1 & -1 & 5
\end{array}\right]
$$

The density matrix can then be reconstructed linearly as $\rho=\sum_{\left\{a_{1}, a_{2}\right\}} P\left(a_{1}, a_{2}\right) \mathcal{Q}_{a_{1}, a_{2}}$, with operators $\mathcal{Q}_{a_{1}, a_{2}}=\sum_{\left\{a_{1}^{\prime}, a_{2}^{\prime}\right\}}\left(T_{a_{1}, a_{1}^{\prime}}^{-1} \otimes T_{a_{2}, a_{2}^{\prime}}^{-1}\right)\left(M_{a_{1}^{\prime}} \otimes M_{a_{2}^{\prime}}\right)$.

Furthermore, expectation values of general operators $\mathcal{O}$ can be rewritten in terms of the probability distribution $P\left(a_{1}, a_{2}\right)$,

$$
\begin{aligned}
\langle\mathcal{O}\rangle & =\operatorname{Tr}[\rho \mathcal{O}] \\
& =\sum_{\left\{a_{1}, a_{2}\right\}} Q_{a_{1}, a_{2}}^{\mathcal{O}} P\left(a_{1}, a_{2}\right),
\end{aligned}
$$

with $Q_{a_{1}, a_{2}}^{\mathcal{O}}=\sum_{\left\{a_{1}^{\prime}, a_{2}^{\prime}\right\}} \operatorname{Tr}\left[\mathcal{O} M_{a_{1}^{\prime}} \otimes M_{a_{2}^{\prime}}\right] T_{a_{1}, a_{1}^{\prime}}^{-1} \otimes T_{a_{2}, a_{2}^{\prime}}^{-1}$. This enables an efficient evaluation of expectation values by sampling configurations from $P\left(a_{1}, a_{2}\right)$ in the POVM representation, where the density matrix does not need to be calculated explicitly. The POVM representations of important classes of quantum states can be approximated well and in a scalable way by generative modelling approaches [15]. The computational bottleneck of these methods is the generation of samples from the model distribution, and can potentially be alleviated using neuromorphic devices.

The Bell state is encoded in a sampling spiking network as follows. The visible neurons $\boldsymbol{v}$ are identified with the qubits $\boldsymbol{a}$ in the POVM representation. The network parameters are trained such that the distribution $P_{\mathrm{B}}\left(a_{1}, a_{2}\right)$ is represented by the network. To achieve this, we need to translate the variables $a_{1}, a_{2}$, which can take four possible values each, into binary neurons $\boldsymbol{v}$, where each neuron can take the values 0 or 1 . The mapping to four binary visible neurons $v_{1}, \ldots, v_{4}$ is accomplished by defining

$$
a_{1}=2 v_{1}+v_{2}, \quad a_{2}=2 v_{3}+v_{4} .
$$

From this we can derive the distribution $p_{\mathrm{B}}^{*}(\boldsymbol{v})$ over the states of the visible neurons and have all ingredients to encode the Bell state in our spiking network. 
Analogously, the probability distribution for the two-qubit Werner state with noise contribution $r$ can be derived from its density matrix 23, 39],

$$
\rho_{\mathrm{W}}=\frac{1}{4}\left[\begin{array}{cccc}
1+r & 0 & 0 & 2 r \\
0 & 1-r & 0 & 0 \\
0 & 0 & 1-r & 0 \\
2 r & 0 & 0 & 1+r
\end{array}\right] .
$$

The same is true for Greenberger-Horne-Zeilinger (GHZ) states of more than two qubits, described by the density matrices [24,

$$
\rho_{\mathrm{GHZ}}=\frac{1}{2}\left[\begin{array}{ccccc}
1 & 0 & \ldots & 0 & 1 \\
0 & & & & 0 \\
\vdots & & 0 & & \vdots \\
0 & & & & 0 \\
1 & 0 & \ldots & 0 & 1
\end{array}\right] .
$$

We can then approximate the corresponding probability distributions by a spiking sampling network.

\section{Training algorithm}

Our goal is to approximate a target distribution $p^{*}(\boldsymbol{v})$ by the model distribution $p(\boldsymbol{v} ; \mathcal{W})$ encoded by the spiking neuromorphic hardware. The distance between the two distributions is quantified by the Kullback-Leibler divergence,

$$
\begin{aligned}
D_{\mathrm{KL}}\left(p^{*} \| p\right)= & \sum_{\{\boldsymbol{v}\}} p^{*}(\boldsymbol{v}) \ln \left[\frac{p^{*}(\boldsymbol{v})}{p(\boldsymbol{v} ; \mathcal{W})}\right] \\
= & \sum_{\{\boldsymbol{v}\}} p^{*}(\boldsymbol{v})\left(\ln \left[p^{*}(\boldsymbol{v})\right]-\ln [\tilde{p}(\boldsymbol{v} ; \mathcal{W})]\right. \\
& +\ln [Z(\mathcal{W})]) .
\end{aligned}
$$

Here we assumed that $p(\boldsymbol{v} ; \mathcal{W})$ is well described by the marginal of a Boltzmann distribution and introduced the unnormalized probability distribution $\tilde{p}(\boldsymbol{v} ; \mathcal{W})=\sum_{\{\boldsymbol{h}\}} \exp [-E(\boldsymbol{v}, \boldsymbol{h} ; \mathcal{W})]$ as the exponential of the negative network energy, as well as the partition sum $Z(\mathcal{W})=$ $\sum_{\{\boldsymbol{v}, \boldsymbol{h}\}} \exp [-E(\boldsymbol{v}, \boldsymbol{h} ; \mathcal{W})]$, which allows us to replace $p(\boldsymbol{v} ; \mathcal{W})=\tilde{p}(\boldsymbol{v} ; \mathcal{W}) / Z(\mathcal{W})$ in the second line of eq. (13).

The gradient of the Kullback-Leibler divergence with respect to a general connecting 
weight $W_{i, j}$ is given by

$$
\begin{aligned}
& \frac{\partial D_{\mathrm{KL}}}{\partial W_{i, j}}=\sum_{\{\boldsymbol{v}\}} p^{*}(\boldsymbol{v})\left[-\frac{1}{\tilde{p}(\boldsymbol{v} ; \mathcal{W})} \frac{\partial \tilde{p}(\boldsymbol{v} ; \mathcal{W})}{\partial W_{i, j}}\right. \\
& \left.+\frac{1}{Z(\mathcal{W})} \frac{\partial Z(\mathcal{W})}{\partial W_{i, j}}\right] \\
& =\sum_{\{\boldsymbol{v}\}} p^{*}(\boldsymbol{v})\left[-\frac{1}{\tilde{p}(\boldsymbol{v} ; \mathcal{W})}\left(\sum_{\{\boldsymbol{h}\}} v_{i} h_{j} e^{-E(\boldsymbol{v}, \boldsymbol{h} ; \mathcal{W})}\right)\right. \\
& \left.+\frac{1}{Z(\mathcal{W})}\left(\sum_{\left\{\boldsymbol{v}^{\prime}, \boldsymbol{h}\right\}} v_{i}^{\prime} h_{j} e^{-E\left(\boldsymbol{v}^{\prime}, \boldsymbol{h} ; \mathcal{W}\right)}\right)\right] \\
& =-\sum_{\{\boldsymbol{v}, \boldsymbol{h}\}} \frac{p^{*}(\boldsymbol{v})}{p(\boldsymbol{v} ; \mathcal{W})} v_{i} h_{j} \frac{e^{-E(\boldsymbol{v}, \boldsymbol{h} ; \mathcal{W})}}{Z(\mathcal{W})} \\
& +\sum_{\left\{\boldsymbol{v}^{\prime}, \boldsymbol{h}\right\}} v_{i}^{\prime} h_{j} \frac{e^{-E\left(\boldsymbol{v}^{\prime}, \boldsymbol{h} ; \mathcal{W}\right)}}{Z(\mathcal{W})} \\
& =\sum_{\{\boldsymbol{v}, \boldsymbol{h}\}}\left[1-\frac{p^{*}(\boldsymbol{v})}{p(\boldsymbol{v} ; \mathcal{W})}\right] v_{i} h_{j} \frac{\exp [-E(\boldsymbol{v}, \boldsymbol{h} ; \mathcal{W})]}{Z(\mathcal{W})} \\
& =\left\langle\left[1-\frac{p^{*}(\boldsymbol{v})}{p(\boldsymbol{v} ; \mathcal{W})}\right] v_{i} h_{j}\right\rangle_{p(\boldsymbol{v}, \boldsymbol{h} ; \mathcal{W})} .
\end{aligned}
$$

Thus, the weight updates are calculated by drawing a sample set of network states, evaluating the probability $p(\boldsymbol{v} ; \mathcal{W})$ underlying the configurations in the set, and calculating the expectation value of the product of the two connected neurons, weighted with $1-p^{*}(\boldsymbol{v}) / p(\boldsymbol{v} ; \mathcal{W})$. When using the spiking network on the BrainScaleS-2 system, we draw these sample states by observing the network at regular points in time spaced by $2 \mu \mathrm{s}$ (for a refractory time of about $10 \mu \mathrm{s}$, see App. A.

The weight update in training epoch $t$ then reads

$$
W_{i, j}^{t}=W_{i, j}^{t-1}-\eta\left\langle\left[1-\frac{p^{*}(\boldsymbol{v})}{p(\boldsymbol{v} ; \mathcal{W})}\right] v_{i} h_{j}\right\rangle_{p(\boldsymbol{v}, \boldsymbol{h} ; \mathcal{W})},
$$

with learning rate $\eta$. Analogously, updates for the biases can be derived,

$$
\begin{aligned}
& b_{j}^{t}=b_{j}^{t-1}-\eta\left\langle\left[1-\frac{p^{*}(\boldsymbol{v})}{p(\boldsymbol{v} ; \mathcal{W})}\right] h_{j}\right\rangle_{p(\boldsymbol{v}, \boldsymbol{h} ; \mathcal{W})}, \\
& d_{i}^{t}=d_{i}^{t-1}-\eta\left\langle\left[1-\frac{p^{*}(\boldsymbol{v})}{p(\boldsymbol{v} ; \mathcal{W})}\right] v_{i}\right\rangle_{p(\boldsymbol{v}, \boldsymbol{h} ; \mathcal{W})} .
\end{aligned}
$$

If connections between the visible neurons exist in the network structure, the updates for those connecting weights are analogous to Eq. (15), where the weighted expectation value of the product of the corresponding visible neurons is evaluated. This learning scheme is a modified version of wake-sleep learning [13].

Since this training algorithm is based on a gradient-descent ansatz, we can apply further modifications which lead to better convergence, such as a momentum approach to avoid 
getting stuck at a local minimum. In our simulations, we apply the Adam optimizer scheme. This scheme combines a momentum approach with an adaptive learning rate which is chosen for each network parameter individually. The update for a general network parameter $\mathcal{W}_{k}$ is given, in the Adam optimizer, by

$$
\begin{aligned}
m_{k}^{t} & =\beta_{1} m_{k}^{t-1}+\left(1-\beta_{1}\right) \frac{\partial D_{\mathrm{KL}}\left(p^{*} \| p\right)}{\partial \mathcal{W}_{k}}, \\
v_{k}^{t} & =\beta_{2} v_{k}^{t-1}+\left(1-\beta_{2}\right)\left[\frac{\partial D_{\mathrm{KL}}\left(p^{*} \| p\right)}{\partial \mathcal{W}_{k}}\right]^{2}, \\
\hat{m}_{k}^{t} & =\frac{m_{k}^{t}}{1-\beta_{1}^{t}}, \quad \hat{v}_{k}^{t}=\frac{v_{k}^{t}}{1-\beta_{2}^{t}}, \\
\mathcal{W}_{k}^{t} & =\mathcal{W}_{k}^{t-1}-\eta \frac{\hat{m}_{k}^{t}}{\sqrt{\hat{v}_{k}^{t}}+\varepsilon},
\end{aligned}
$$

where $m_{k}$ acts as a momentum and $v_{k}$ sets the adaptive learning rate. Here we follow the common choice and set the hyper-parameters to $\beta_{1}=0.9, \beta_{2}=0.999$, and $\varepsilon=10^{-8}$, [40]. We additionally multiply the adaptive learning rate with an exponentially decaying factor $\eta(t)$ from an initial value of $\eta_{\text {init }}=1$ to a minimum value of $\eta_{\min }=0.001$,

$$
\eta(t)=\max \left(\eta_{\text {init }} \exp [-0.001 t], \eta_{\min }\right),
$$

where $t$ counts the training epochs. Note that this learning rate is a hyper-parameter that needs to be chosen accordingly and requires a special form for the discrete-valued weights and biases on the neuromorphic hardware. With the exponentially decaying factor we ensure that the learning rate is large enough to cause changes in the weights at short time scales, but is small enough to enables convergence at later times.

In general, Hebbian training algorithms are based on minimizing the correlation mismatch between data and model distributions. The traditional way for estimating this mismatch is contrastive divergence [13,41], where the target and model distributions are approximated by a single layer-wise network update (CD-1). This method can only be used to obtain an update of the network parameters for layer-wise connected networks and essentially represents a performance optimization with respect to sampling from the complete distributions. For physical neuromorphic systems, the notion of a "single network update" becomes meaningless and the performance characteristics make the actual sampling run cheaply as compared with the start-up cost. We take advantage of this difference by using the full model distribution for calculating updates. We thus reconstruct the target visible distribution from the model distribution by reweighting as described above. The advantage of this strategy is that arbitrary network architectures including partially restricted and deep networks can be used.

\section{References}

[1] C. S. Thakur, J. L. Molin, G. Cauwenberghs, G. Indiveri, K. Kumar, N. Qiao, J. Schemmel, R. Wang, E. Chicca, J. Olson Hasler, J. Seo, S. Yu et al., Large-scale neuromorphic spiking array processors: A quest to mimic the brain, Front. Neurosci. 12, 891 (2018), doi:10.3389/fnins.2018.00891. 
[2] K. Roy, A. Jaiswal and P. Panda, Towards spike-based machine intelligence with neuromorphic computing, Nature 575(7784), 607 (2019), doi:10.1038/s41586-019-1677-2.

[3] M. Davies, N. Srinivasa, T.-H. Lin, G. Chinya, Y. Cao, S. H. Choday, G. Dimou, P. Joshi, N. Imam, S. Jain et al., Loihi: A neuromorphic manycore processor with on-chip learning, IEEE Micro 38(1), 82 (2018), doi:10.1109/MM.2018.112130359.

[4] P. A. Merolla, J. V. Arthur, R. Alvarez-Icaza, A. S. Cassidy, J. Sawada, F. Akopyan, B. L. Jackson, N. Imam, C. Guo, Y. Nakamura et al., A million spiking-neuron integrated circuit with a scalable communication network and interface, Science 345(6197), 668 (2014), doi:10.1126/science.1254642.

[5] J. Pei, L. Deng, S. Song, M. Zhao, Y. Zhang, S. Wu, G. Wang, Z. Zou, Z. Wu, W. He et al., Towards artificial general intelligence with hybrid Tianjic chip architecture, Nature 572(7767), 106 (2019), doi:10.1038/s41586-019-1424-8.

[6] F. Cai, J. M. Correll, S. H. Lee, Y. Lim, V. Bothra, Z. Zhang, M. P. Flynn and W. D. Lu, A fully integrated reprogrammable memristor-cmos system for efficient multiplyaccumulate operations, Nature Electronics 2(7), 290 (2019), doi:10.1038/s41928-0190270-x.

[7] I. Boybat, M. Le Gallo, S. Nandakumar, T. Moraitis, T. Parnell, T. Tuma, B. Rajendran, Y. Leblebici, A. Sebastian and E. Eleftheriou, Neuromorphic computing with multimemristive synapses, Nature communications 9(1), 1 (2018), doi:10.1038/s41467-01804933-y.

[8] S. Moradi, N. Qiao, F. Stefanini and G. Indiveri, A scalable multicore architecture with heterogeneous memory structures for dynamic neuromorphic asynchronous processors (DYNAPs), IEEE transactions on biomedical circuits and systems 12(1), 106 (2017), doi:10.1109/TBCAS.2017.2759700.

[9] S. B. Furber, F. Galluppi, S. Temple and L. A. Plana, The Spinnaker project, Proceedings of the IEEE 102(5), 652 (2014), doi:10.1109/JPROC.2014.2304638.

[10] M. A. Petrovici, S. Schmitt, J. Klähn, D. Stöckel, A. Schroeder, G. Bellec, J. Bill, O. Breitwieser, I. Bytschok, A. Grübl et al., Pattern representation and recognition with accelerated analog neuromorphic systems, In 2017 IEEE International Symposium on Circuits and Systems (ISCAS), pp. 1-4. IEEE, doi:10.1109/ISCAS.2017.8050530 (2017).

[11] S. Billaudelle, Y. Stradmann, K. Schreiber, B. Cramer, A. Baumbach, D. Dold, J. Göltz, A. F. Kungl, T. C. Wunderlich, A. Hartel et al., Versatile emulation of spiking neural networks on an accelerated neuromorphic substrate, In 2020 IEEE International Symposium on Circuits and Systems (ISCAS), pp. 1-5. IEEE, doi:10.1109/ISCAS45731.2020.9180741 (2020).

[12] R. P. Feynman, Simulating physics with computers, Int. J. Theor. Phys. 21(6), 467 (1982), doi:10.1007/BF02650179.

[13] G. Hinton, P. Dayan, B. Frey and R. Neal, The "wake-sleep" algorithm for unsupervised neural networks, Science 268(5214), 1158 (1995), doi:10.1126/science.7761831. 
[14] A. Peres, Quantum Theory: Concepts and Methods, Springer, Dordrecht, ISBN 0-79233632-1, doi:10.1007/0-306-47120-5 (2002).

[15] J. Carrasquilla, G. Torlai, R. G. Melko and L. Aolita, Reconstructing quantum states with generative models, Nat. Mach. Intell. 1(3), 155 (2019), doi:10.1038/s42256-019-0028-1.

[16] D. Dold, I. Bytschok, A. F. Kungl, A. Baumbach, O. Breitwieser, W. Senn, J. Schemmel, K. Meier and M. A. Petrovici, Stochasticity from function-why the bayesian brain may need no noise, Neural Networks 119, 200 (2019), doi:10.1016/j.neunet.2019.08.002.

[17] A. F. Kungl, S. Schmitt, J. Klähn, P. Müller, A. Baumbach, D. Dold, A. Kugele, E. Müller, C. Koke, M. Kleider, C. Mauch, O. Breitwieser et al., Accelerated physical emulation of bayesian inference in spiking neural networks, Front. Neurosci. 13, 1201 (2019), doi:10.3389/fnins.2019.01201.

[18] M. A. Petrovici, J. Bill, I. Bytschok, J. Schemmel and K. Meier, Stochastic inference with spiking neurons in the high-conductance state, Phys. Rev. E 94, 042312 (2016), doi:10.1103/PhysRevE.94.042312.

[19] M. A. Petrovici, Form Versus Function: Theory and Models for Neuronal Substrates, Springer International Publishing, doi:10.1007/978-3-319-39552-4 (2016).

[20] J. S. Bell, Speakable and Unspeakable in Quantum Mechanics: Collected Papers on Quantum Philosophy, Cambridge University Press, 2 edn., doi:10.1017/CBO9780511815676 (2004).

[21] J. F. Clauser, M. A. Horne, A. Shimony and R. A. Holt, Proposed experiment to test local hidden-variable theories, Phys. Rev. Lett. 23, 880 (1969), doi:10.1103/PhysRevLett.23.880.

[22] A. Aspect, P. Grangier and G. Roger, Experimental tests of realistic local theories via Bell's theorem, Phys. Rev. Lett. 47, 460 (1981), doi:10.1103/PhysRevLett.47.460.

[23] A. Cabello, A. Feito and A. Lamas-Linares, Bell's inequalities with realistic noise for polarization-entangled photons, Phys. Rev. A 72, 052112 (2005), doi:10.1103/PhysRevA.72.052112.

[24] D. M. Greenberger, M. A. Horne and A. Zeilinger, Going Beyond Bell's Theorem, pp. 69-72, Springer Netherlands, Dordrecht, ISBN 978-94-017-0849-4, doi:10.1007/978-94017-0849-4_10 (1989).

[25] D. Leibfried, E. Knill, S. Seidelin, J. Britton, R. B. Blakestad, J. Chiaverini, D. B. Hume, W. M. Itano, J. D. Jost, C. Langer, R. Ozeri, R. Reichle et al., Creation of a six-atom 'Schrödinger cat' state, Nature 438(7068), 639 (2005), doi:10.1038/nature04251.

[26] N. Le Roux and Y. Bengio, Representational power of restricted Boltzmann machines and deep belief networks, Neural Comput. 20(6), 1631 (2008), doi:10.1162/neco.2008.0407-510.

[27] T. Wunderlich, A. F. Kungl, E. Müller, A. Hartel, Y. Stradmann, S. A. Aamir, A. Grübl, A. Heimbrecht, K. Schreiber, D. Stöckel, C. Pehle, S. Billaudelle et al., Demonstrating advantages of neuromorphic computation: A pilot study, Front. Neurosci. 13, 260 (2019), doi: $10.3389 /$ fnins.2019.00260. 
[28] S. Aaronson, The learnability of quantum states, P. Roy. Soc. A-Math. Phy. 463(2088), 3089 (2007), doi:10.1098/rspa.2007.0113.

[29] G. Carleo and M. Troyer, Solving the quantum many-body problem with artificial neural networks, Science 355(6325), 602 (2017), doi:10.1126/science.aag2302.

[30] R. G. Melko, G. Carleo, J. Carrsquilla and J. I. Cirac, Restricted Boltzmann machines in quantum physics, Nat. Phys. 15(9), 887 (2019), doi:10.1038/s41567-019-0545-1.

[31] C. Wetterich, Quantum computing with classical bits, Nucl. Phys. B 948, 114776 (2019), doi:10.1016/j.nuclphysb.2019.114776.

[32] S. Czischek, J. M. Pawlowski, T. Gasenzer and M. Gärttner, Sampling scheme for neuromorphic simulation of entangled quantum systems, Phys. Rev. B 100, 195120 (2019), doi:10.1103/PhysRevB.100.195120.

[33] L. Kades and J. M. Pawlowski, Discrete Langevin machine: Bridging the gap between thermodynamic and neuromorphic systems, Phys. Rev. E 101, 063304 (2020), doi:10.1103/PhysRevE.101.063304.

[34] I. Bytschok, D. Dold, J. Schemmel, K. Meier and M. A. Petrovici, Spike-based probabilistic inference with correlated noise, arXiv:1707.01746 (2017).

[35] O. Breitwieser, A. Baumbach, A. Korcsak-Gorzo, J. Klähn, M. Brixner and M. Petrovici, sbs: Spike-based sampling (v1.8.2), doi:10.5281/zenodo.3686015 (2020).

[36] D. von Suchodoletz, B. Wiebelt, K. Meier and M. Janczyk, Flexible hpc: bwforcluster nemo, Proceedings of the 3rd bwHPCSymposium: Heidelberg (2016).

[37] B. Cramer, S. Billaudelle, S. Kanya, A. Leibfried, A. Grübl, V. Karasenko, C. Pehle, K. Schreiber, Y. Stradmann, J. Weis et al., Training spiking multi-layer networks with surrogate gradients on an analog neuromorphic substrate, arXiv:2006.07239[cs.NE] (2020).

[38] J. Göltz, A. Baumbach, S. Billaudelle, O. Breitwieser, D. Dold, L. Kriener, A. F. Kungl, W. Senn, J. Schemmel, K. Meier et al., Fast and deep neuromorphic learning with timeto-first-spike coding, arXiv:1912.11443[cs.NE] (2019).

[39] R. F. Werner, Quantum states with Einstein-Podolsky-Rosen correlations admitting a hidden-variable model, Phys. Rev. A 40, 4277 (1989), doi:10.1103/PhysRevA.40.4277.

[40] D. P. Kingma and J. Ba, Adam: A method for stochastic optimization, arXiv:1412.6980 [cs.LG] (2014).

[41] G. E. Hinton, Training products of experts by minimizing contrastive divergence, Neural Comput. 14(8), 1771 (2002), doi:10.1162/089976602760128018. 